\title{
Parathyroidectomy improves cardiovascular risk factors in normocalcemic and hypercalcemic primary hyperparathyroidism
}

Selvihan Beysel ${ }^{1,2,3^{*}}$ (D, Mustafa Caliskan ${ }^{1}$, Muhammed Kizilgul', Mahmut Apaydin', Seyfullah Kan', Mustafa Ozbek ${ }^{1}$ and Erman Cakal ${ }^{1}$

\begin{abstract}
Background: Parathyroidectomy has ameliorated cardiovascular risk factors in patients with hypercalcemic primary hyperparathyroidism (PHPT), but the effect of parathyroidectomy on normocalcemic PHPT is not exactly known. This case-controlled study aimed to investigate the effect of parathyroidectomy on cardiovascular risk factors in patients with normocalcemic and hypercalcemic PHPT.

Methods: Subjects with normocalcemic PHPT $(n=35)$, age- and sex-matched hypercalcemic PHPT $(n=60)$ and age- and sex-matched control $(n=60)$ were included. Cardiometabolic disorders were investigated with traditional cardiometabolic risk factors and the Framingham cardiovascular risk score (CRS) before and 6 months after parathyroidectomy.

Results: Diabetes, dyslipidemia, hypertension, obesity, insulin resistance, osteoporosis, having fractures were similarly increased in the hypercalcemic and normocalcemic PHPT groups $(p>0.05)$ compared with the controls $(p$ $<0.05$ ). Blood pressures, glucose metabolism (glucose, insulin, HOMA-IR) and lipid profiles were similarly increased in the PHPT groups $(p>0.05)$ compared with the controls $(p<0.05)$. After parathyroidectomy, blood pressures, serum total cholesterol, and HOMA-IR were decreased in both PHPT groups $(p<0.05)$. CRS was lower in the controls $(5.74 \pm 3.24, p<0.05)$. After parathyroidectomy, CRS was decreased in the normocalcemic $(11.98 \pm 10.11$ vs. $7.37 \pm 4.48)$ and hypercalcemic (14.62 \pm 11.06 vs. $8.05 \pm 7.72)$ PHPT groups. Increased blood pressures were independent predictors of serum $\mathrm{PPTH}$.

Conclusion: The normocalcemic and hypercalcemic PHPT groups had similarly increased cardiovascular risk factors, even independently of serum calcium. Parathyroidectomy ameliorated the increased cardiovascular risk factors in both normocalcemic and hypercalcemic PHPT.
\end{abstract}

Keywords: Normocalcemic primary hyperparathyroidism, Hypercalcemic primary hyperparathyroidism, Cardiovascular risk

\footnotetext{
* Correspondence: beyselselvihan@gmail.com

${ }^{1}$ Department of Endocrinology and Metabolism, Ankara Diskapi Yildirim

Beyazit Teaching and Research Hospital, Ankara, Turkey

2Department of Medical Biology, Baskent University, Ankara, Turkey

Full list of author information is available at the end of the article
}

(c) The Author(s). 2019 Open Access This article is distributed under the terms of the Creative Commons Attribution 4.0 International License (http://creativecommons.org/licenses/by/4.0/) which permits unrestricted use, distribution, and reproduction in any medium, provided you give appropriate credit to the original author(s) and the source, provide a link to the Creative Commons license, and indicate if changes were made. The Creative Commons Public Domain Dedication waiver (http://creativecommons.org/publicdomain/zero/1.0/) applies to the data made available in this article, unless otherwise stated. 


\section{Background}

Primary hyperparathyroidism (PHPT) is characterized by hypercalcemia and elevated parathyroid hormone $(\mathrm{PTH})$ concentrations. Normocalcemic PHPT represents a special form of PHPT, with normal total serum calcium and ionized calcium concentrations in the presence of elevated PTH concentrations $[1,2]$. In such cases, the following secondary conditions that cause elevated PTH should be ruled out: renal disease, vitamin D deficiency, renal hypercalciuria, calcium malabsorption, and thiazide or lithium usage [3]. Typically, normocalcemic patients with PHPT present with symptomatic complications such as osteoporosis and nephrolithiasis. Non-traditional manifestations of normocalcemic PHPT, namely cardiovascular and metabolic disorders, are not known by physicians who recognize PHPT by its symptomatic complications. The asymptomatic form of normocalcemic PHPT has also been defined in population-based screening programs [4, 5]. Hypercalcemic PHPT is associated with increased hypertension, dyslipidemia, obesity, impaired glucose intolerance, and diabetes mellitus [6, 7], as well as cardiovascular morbidity and mortality[1, 8-11]. Parathyroidectomy ameliorates those cardiovascular risk factors in patients with hypercalcemic PHPT [7-11]. However, the effect of parathyroidectomy on cardiovascular risk factors in patients with normocalcemic PHPT is not exactly known. In population-based studies, elevated calcium and PTH concentrations have been linked to increased cardiovascular risk [12-15]. There is lack of data on the effect of parathyroidectomy on cardiovascular risk factors in patients with normocalcemic PHPT [16]. This study aimed to investigate the effect of parathyroidectomy on cardiometabolic disorders in patients with normocalcemic and hypercalcemic PHPT.

\section{Methods}

\section{Patients}

Patients with PHPT treated in the Department of Endocrinology and Metabolism, Ankara Diskapi Training and Research Hospital, from 2013 to 2017, were recruited to this case-control study. Normocalcemic patients with PHPT $(n=35)$ and age- and sex-matched patients with hypercalcemic PHPT $(n=60)$, and age- and sex-matched control subjects $(n=60)$ were included. The controls subjects were selected from population-based screening programs. Controls subjects without diabetes and hyperparathyroidism were included. Subjects using anti-lipidemic drugs and antihypertensive drugs were not included. Most patients with normocalcemia were referred from the osteoporosis outpatient clinic.

In our report, 54 patients had normocalcemia with elevated PTH concentrations, and 19 patients who were normocalcemic and treated with bisphosphonates were excluded form study. Forty-two patients who were hypercalcemic and treated with bisphosphonates were excluded. Patients with hypercalcemic $(n=60)$ and normocalcemic PHPT $(n=35)$ who were not using bisphosphonates were included. We evaluated symptoms related with hypercalcemia (repeated nephrolithiasis, gastritis, polyuria, muscle weakness, osteoporosis or psychiatric disorders) and bone fractures.

Hypercalcemic crisis was defined as serum calcium $\geq 14 \mathrm{mg} / \mathrm{dL}$. Patients with hypercalcemic crisis were not included. The diagnosis of hypercalcemic PHPT was based on elevated serum albumin-corrected calcium (> $10.5 \mathrm{mg} / \mathrm{dL}$ ) with high serum iPTH concentrations ( $>65$ $\mathrm{pg} / \mathrm{mL}$ ). Normocalcemic PHPT was diagnosed in the presence of elevated serum iPTH concentrations $(>65$ $\mathrm{pg} / \mathrm{mL}$ ) with normal serum albumin-corrected calcium $(\leq 10.4 \mathrm{mg} / \mathrm{dL})$ on two separate occasions. All patients had 25-hydroxyvitamin D $(25(\mathrm{OH})$ vitamin D) concentrations above $30 \mathrm{ng} / \mathrm{mL}$.

Secondary causes of hyperparathyroidism were excluded, including renal insufficiency (creatinine clearance $<90 \mathrm{~mL} /$ $\mathrm{min}$ ), liver disease, significant hypercalciuria (urinary calcium $>350 \mathrm{mg}$ per $24 \mathrm{~h}$ ), thiazide or lithium use and other metabolic bone diseases. All patients with PHPT underwent localization studies such as neck ultrasonography and Tc99-sestamibi and/or single-photon emission-computed tomography (SPECT-CT) scintigraphy. All patients with PHPT underwent four gland explorations and the incision size was the same in the two groups. All operations were performed by a single surgical group. All patients had the following characteristics: biochemical and clinical diagnosis of PHPT, parathyroidectomy, successful parathyroidectomy as confirmed by normal post-parathyroidectomy serum calcium and iPTH concentrations and pathologic confirmation of PHPT. Patients with incomplete clinical and biochemical evaluation were eliminated. Diabetes, dyslipidemia, hypertension, obesity, fractures, smoking and insulin resistance were recorded. Medications including anti-lipidemics, antihypertensives, and oral antidiabetics/insulin were recorded. Each subject gave written informed consent in accordance with the Declaration of Helsinki, and this study was approved by Diskapi Teaching and Training Hospital Ethic Committee.

\section{Measurements}

Cardiometabolic disorders were investigated through traditional cardiometabolic risk factors and the Framingham 10-year general cardiovascular risk score before and after parathyroidectomy. Anthropometric measurements and biochemical analysis were performed in all patients before surgery and 6 months after surgery. The waist-hip ratio and body mass index (BMI) was calculated. Office blood pressure (BP) was measured in each subject in the sitting position after $5 \mathrm{~min}$ of rest, provided that the arm was supported at the level of the heart. A cuff covered 
about $80 \%$ of the circumference of the upper arm with the lower edge $2.5-3 \mathrm{~cm}$ above the elbow. Hypertension was confirmed by repeated measurements of systolic blood pressure (BP) $>140 \mathrm{mmHg}$ and diastolic $\mathrm{BP}>90 \mathrm{mmHg}$ on two separate occasions. Serum calcium, phosphorus, intact parathyroid hormone (iPTH), 25( $\mathrm{OH})$ vitamin $\mathrm{D}$, alkaline phosphatase (ALP), glycated hemoglobin (HbA1c), total cholesterol, high-density lipoprotein cholesterol (HDL-C), low-density lipoprotein cholesterol (LDL-C), triglycerides (TG), glucose, and insulin were measured after overnight fasting. Patients performed a 24-hour urine collection for measurements of urinary calcium excretion. iPTH was measured using a radioimmunoassay method (DiaSorin Inc., Stillwater, USA). Serum total calcium was corrected for albumin using the following formula:

$$
\begin{aligned}
& \text { albumin-corrected calcium } \\
& \quad=[0.8 \times(4-\text { serum albumin })]+\text { serum calcium }
\end{aligned}
$$

Diabetes and prediabetes were diagnosed as follows: diabetes HbA1c $6.5 \%$ or higher; prediabetes HbA1c 5.7 to $6.4 \%$; normal less than $\mathrm{HbA1c} 5.7 \%$. Insulin resistance was calculated using the homeostasis model assessment -insulin resistance (HOMA-IR) formula [17]:

[fasting plasma insulin $(\mu \mathrm{IU} / \mathrm{ml}) \mathrm{X}$ fasting serum glucose $(\mathrm{mg} / \mathrm{dl})] / 405$

The Framingham General Cardiovascular Risk Score (10-year risk) (CRS) was computed through the online interactive risk score calculator available on the Framingham Heart Study website [18]. The 10-year general $\mathrm{CV}$ risk profile based on the Framingham study consisted of a total of 7 items such as sex, age, systolic BP, HDL-C, total cholesterol, and whether the individual had diabetes or smoked.

\section{Statistical analysis}

Statistical analysis was performed using the SPSS 18.0 (SPSS, Inc.) statistical software. Variables are presented as mean \pm standard deviation (SD) or median (with interquartile range), percentage (\%), odds ratios (OR) and $95 \%$ confidence intervals $(C I)$. Normality was tested using the Kolmogorov-Smirnov and Shapiro-Wilk $W$ test. The Chi-square test or Fisher's exact test, where appropriate, was used for categorical variables. Student's t-test was used for the analysis of continuous variables between the two groups. The paired-sample t-test was used for the two groups before and after parathyroidectomy. Backward logistic regression was performed: serum iPTH was defined as dependent variable and calcium, LDL-C, HOMA-IR, creatinine clearance, and systolic and diastolic BP were independent variables. Statistical significance was defined as a $p<0.05$.

\section{Results}

Sex and mean age were similar between the groups $(p>$ $0.05)$. Some of the controls were newly diagnosed as having dyslipidemia (16.7\%) and hypertension (15.0\%) in population-based screening programs during the study. The rate of prediabetes was $8.7 \%$ among the controls according to HbA1c levels. The prevalence of patients with diabetes, dyslipidemia, hypertension, obesity, insulin resistance, osteoporosis, having fractures, and medications usage were similar between hypercalcemic and normocalcemic PHPT groups $(p>0.05)$. Serum calcium and iPTH concentrations and urinary calcium excretion were higher in patients with hypercalcemic PHPT compared with normocalcemic PHPT $(p<0.05)$. Blood pressures and creatinine clearance were similar between the PHPT groups $(p>0.05)$. Glucose metabolism (glucose, insulin, HOMA-IR) and lipid profiles were similar between the PHPT groups $(p>0.05)$. Cardiovascular risk score was lower in controls, but similar between the PHPT groups $(5.74 \pm 3.24,11.98 \pm 10.11$, and $14.62 \pm 11.07$, respectively). The baseline characteristics of the groups before parathyroidectomy are shown in Table 1.

After parathyroidectomy, serum calcium, iPTH, and ALP concentrations decreased, whereas serum phosphorous was increased in both PHPT groups $(p<0.05)$. Systolic and diastolic blood pressure was decreased in both PHPT groups $(p<0.05)$. Serum total cholesterol and HOMA-IR was decreased in both PHPT groups $(p$ $<0.05)$. The change in metabolic parameters before and after parathyroidectomy is shown in Table 2. Cardiovascular risk score was decreased after parathyroidectomy in the normocalcemic $(11.98 \pm 10.11$ vs. $7.37 \pm 4.48)$ and hypercalcemic (14.62 \pm 11.06 vs. $8.05 \pm 7.72)$ PHPT groups. After parathyroidectomy, patients with fractures had more severe cardiovascular risk score compared with patients without fractures $(6.72 \pm 4.92$ vs. $14.75 \pm 9.47, p=0.010)$ (Table 3). Hypercalcemic (OR $=4.39,95 \%$ CI: [2.51-8.53]; $p<0.001)$ and normocalcemic $(O R=3.16,95 \% \mathrm{CI}$ : [2.7 8 -6.92]; $p=0.002)$ PHPT was associated with increased in cardiovascular risk score. Systolic blood pressure $(\beta=$ 1.081, 95\% CI: [1.03-1.13]; $p=0.001)$ and diastolic blood pressure $(\beta=1.192,95 \%$ CI: $[1.04-1.32]$; $p=0.05)$ were independent predictors of serum iPTH values, but not associated with calcium values (Cox \& Snell $\left.R^{2}=0.40\right)$.

\section{Discussion}

The normocalcemic and hypercalcemic PHPT groups had similarly increased cardiovascular risk factors including blood pressures, glucose metabolism, and lipid profiles as well as cardiovascular risk scores compared with the controls. Parathyroidectomy improved the increased cardiovascular risk factors including blood pressures, total cholesterol, and HOMA-IR as well as cardiovascular risk scores in patients with normocalcemic and hypercalcemic 
Table 1 Baseline characteristics of subjects in controls, normocalcemic PHPT and hypercalcemic PHPT group

\begin{tabular}{|c|c|c|c|c|c|c|}
\hline Variables & $\begin{array}{l}\text { Controls }(n=60) \\
\mathrm{n}(\%)\end{array}$ & $\begin{array}{l}\text { Normocalcemic PHPT }(n=35) \\
\mathrm{n}(\%)\end{array}$ & $\begin{array}{l}\text { Hypercalcemic PHPT }(n=60) \\
\text { n (\%) }\end{array}$ & $\mathrm{p} 1^{\mathrm{a}}$ & $p 2^{b}$ & $p 3^{c}$ \\
\hline Female & 48 (80.0\%) & 29 (82.9\%) & $47(78.3 \%)$ & 0.759 & 0.815 & 0.652 \\
\hline Diabetes & - & $8(22.8 \%)$ & $14(23.3 \%)$ & - & - & 0.869 \\
\hline Dyslipidemia & $10(16.7 \%)$ & 22 (62.9\%) & $36(60.0 \%)$ & $<0.001$ & $<0.001$ & 0.835 \\
\hline Hypertension & 9 (15.0\%) & 15 (42.9\%) & $38(63.3 \%)$ & $<0.001$ & $<0.001$ & 0.094 \\
\hline Smoking & $10(16.7 \%)$ & $5(14.3 \%)$ & $12(20.0 \%)$ & 0.743 & 0.759 & 0.526 \\
\hline Obesity & $26(43.3 \%)$ & $16(45.7 \%)$ & $32(53.3 \%)$ & 0.810 & 0.339 & 0.473 \\
\hline İnsulin resistance & $13(21.7 \%)$ & $21(60.0 \%)$ & 41 (68.3\%) & 0.002 & 0.001 & 0.454 \\
\hline Nephrolithiasis & $3(0.05 \%)$ & $9(25.7 \%)$ & $29(48.3 \%)$ & 0.001 & $<0.001$ & 0.048 \\
\hline Osteoporosis & - & $8(22.9 \%)$ & $23(38.3 \%)$ & - & - & 0.064 \\
\hline Fractures & - & $2(5.7 \%)$ & $6(10.0 \%)$ & - & - & 0.624 \\
\hline Subjects using medications (\%) & - & & & - & - & \\
\hline Antilipidemic drugs & & $14(40.0 \%)$ & $27(45.0 \%)$ & & & 0.864 \\
\hline Antihypertensive drugs & & $13(37.1 \%)$ & $33(55.0 \%)$ & & & 0.125 \\
\hline Oral antidiabetic/insulin drugs & & $7(20.0 \%)$ & $13(21.6 \%)$ & & & 0.898 \\
\hline Age (year) & $52.49 \pm 8.17$ & $53.06 \pm 8.76$ & $52.11 \pm 10.04$ & 0.204 & 0.320 & 0.998 \\
\hline BMI $\left(\mathrm{kg} / \mathrm{m}^{2}\right)$ & $29.31 \pm 3.59$ & $29.97 \pm 3.73$ & $31.09 \pm 5.17$ & 0.629 & 0.009 & 0.025 \\
\hline Waist-hip-ratio & $0.92 \pm 0.09$ & $0.90 \pm 0.06$ & $0.90 \pm 0.06$ & 0.293 & 0.375 & 0.852 \\
\hline Diastolic BP (mmHg) & $77.5 \pm 5.4$ & $82.3 \pm 5.8$ & $83.2 \pm 7.6$ & 0.001 & $<0.001$ & 0.563 \\
\hline Systolic BP (mmHg) & $121.6 \pm 9.2$ & $136.6 \pm 16.5$ & $138.9 \pm 16.4$ & $<0.001$ & $<0.001$ & 0.545 \\
\hline iPTH (pg/ml) & $46.1 \pm 10.3$ & $145.8 \pm 40.1$ & $188.5 \pm 93.3$ & $<0.001$ & $<0.001$ & 0.010 \\
\hline Albumin-corrected calcium (mg/dl) & $8.78 \pm 0.29$ & $9.85 \pm 0.30$ & $11.25 \pm 0.69$ & $<0.001$ & $<0.001$ & $<0.001$ \\
\hline Phosphorus (mg/dl) & $3.22 \pm 0.44$ & $2.98 \pm 0.48$ & $2.57 \pm 0.41$ & 0.082 & $<0.001$ & 0.064 \\
\hline ALP (IU/L) & $70.09 \pm 14.85$ & $103.51 \pm 40.82$ & $107.00 \pm 44.27$ & $<0.001$ & $<0.001$ & 0.735 \\
\hline $25(\mathrm{OH})$ vitamin $\mathrm{D}_{3}(\mathrm{nmol} / \mathrm{l})$ & $32.94 \pm 4.89$ & $33.43 \pm 3.18$ & $32.06 \pm 4.65$ & 0.845 & 0.692 & 0.601 \\
\hline Glucose (mg/dl) & $86.69 \pm 5.49$ & $93.11 \pm 12.19$ & $95.00 \pm 14.80$ & 0.007 & 0.003 & 0.567 \\
\hline Insulin ( $\mu \mathrm{l} U / \mathrm{ml})$ & $9.18 \pm 3.96$ & $13.26 \pm 6.19$ & $13.33 \pm 5.24$ & 0.002 & $<0.001$ & 0.953 \\
\hline HOMA-IR & $1.98 \pm 0.90$ & $3.06 \pm 1.47$ & $3.13 \pm 1.25$ & $<0.001$ & $<0.001$ & 0.821 \\
\hline Total cholesterol (mg/dl) & $185.60 \pm 21.22$ & $210.09 \pm 39.46$ & $204.80 \pm 37.98$ & 0.002 & 0.01 & 0.564 \\
\hline LDL-C (mg/dl) & $107.80 \pm 17.61$ & $129.20 \pm 33.11$ & $123.54 \pm 30.33$ & 0.001 & 0.01 & 0.451 \\
\hline $\mathrm{HDL}-\mathrm{C}(\mathrm{mg} / \mathrm{dl})$ & $52.80 \pm 7.68$ & $49.17 \pm 10.56$ & $49.34 \pm 11.29$ & 0.104 & 0.136 & 0.945 \\
\hline TG (mg/dl) & $125.00 \pm 51.20$ & $158.60 \pm 61.78$ & $159.57 \pm 67.34$ & 0.016 & 0.018 & 0.951 \\
\hline Creatinine clerance $\left(\mathrm{ml} / \mathrm{min} / 1.73 \mathrm{~m}^{2}\right)$ & $113.92 \pm 10.85$ & $112.89 \pm 27.26$ & $107.15 \pm 28.37$ & 0.834 & 0.198 & 0.394 \\
\hline Urinary calcium (mmol/dl) & . & $218.69 \pm 162.74$ & $438.49 \pm 186.99$ & - & - & 0.010 \\
\hline Cardiovascular risk score & $5.74 \pm 3.24$ & $11.98 \pm 10.11$ & $14.62 \pm 11.06$ & 0.001 & $<0.001$ & 0.251 \\
\hline
\end{tabular}

${ }^{a}$ controls vs normocalcemic

${ }^{b}$ controls vs hypercalcemic

chypercalcemic vs normocalcemic

Data are presented as mean \pm SD

Abbreviations: BP blood pressure, $i P T H$ intact parathyroid hormone, 25(OH) vitamin D 25-hydroxyvitamin D3, HDL-C high-density-lipoprotein cholesterol, LDL-C lowdensity-lipoprotein cholesterol, TG triglycerides, HOMA-IR homestasis model assessment-insulin resistance index, $A L P$ alkaline phosphatase, $P H P T$

primary hyperparathyroidism

Bold represents the significant $p$-values

PHPT. An increased serum PTH concentration was found as a positive predictor of increased blood pressures, even independent of serum calcium.
Normocalcemic PHPT is typically recognized through symptoms such as osteoporosis and nephrolithiasis, and the potential cardiometabolic effects of this disease are 
Table 2 Change in metabolic parameters after parathyroidectomy

\begin{tabular}{|c|c|c|c|c|c|c|}
\hline \multirow[t]{2}{*}{ Variables } & \multicolumn{3}{|c|}{ Normocalcemic PHPT $(n=30)$} & \multicolumn{3}{|c|}{ Hypercalcemic PHPT $(n=50)$} \\
\hline & Pre-PTX & Post-PTX & $\mathrm{P}$ & Pre-PTX & Post-PTX & $P$ \\
\hline BMI $\left(\mathrm{kg} / \mathrm{m}^{2}\right)$ & $29.97 \pm 3.73$ & $29.98 \pm 3.65$ & 0.372 & $31.09 \pm 5.17$ & $29.47 \pm 5.61$ & 0.532 \\
\hline Waist-hip-ratio & $0.89 \pm 0.05$ & $0.88 \pm 0.05$ & 0.177 & $0.89 \pm 0.06$ & $0.88 \pm 0.68$ & 0.363 \\
\hline Diastolic BP (mmHg) & $82.3 \pm 5.8$ & $79.36 \pm 7.74$ & 0.040 & $83.2 \pm 7.6$ & $77.01 \pm 7.38$ & 0.008 \\
\hline Systolic BP (mmHg) & $136.6 \pm 16.5$ & $126.57 \pm 16.57$ & 0.003 & $138.9 \pm 16.4$ & $119.73 \pm 12.95$ & 0.001 \\
\hline iPTH (pg/ml) & $145.8 \pm 40.1$ & $71.52 \pm 21.50$ & $<0.001$ & $188.5 \pm 93.3$ & $48.60 \pm 18.97$ & 0.001 \\
\hline Albumin-corrected calcium (mg/dl) & $9.85 \pm 0.30$ & $8.91 \pm 0.38$ & $<0.001$ & $11.25 \pm 0.69$ & $9.02 \pm 0.32$ & 0.001 \\
\hline Phosphorus (mg/dl) & $2.98 \pm 0.48$ & $3.20 \pm 0.41$ & 0.002 & $2.57 \pm 0.41$ & $3.21 \pm 0.47$ & 0.001 \\
\hline ALP (IU/L) & $103.51 \pm 40.82$ & $86.89 \pm 25.10$ & $<0.001$ & $107.00 \pm 44.27$ & $84.33 \pm 2.54$ & 0.001 \\
\hline Glucose (mg/dl) & $93.11 \pm 12.19$ & $86.36 \pm 5.97$ & 0.120 & $95.00 \pm 14.80$ & $82.26 \pm 6.70$ & 0.009 \\
\hline Insulin $(\mu \mathrm{lU} / \mathrm{ml})$ & $13.26 \pm 6.19$ & $11.47 \pm 3.08$ & 0.006 & $13.33 \pm 5.24$ & $10.83 \pm 4.62$ & 0.125 \\
\hline HOMA-IR & $3.06 \pm 1.47$ & $2.57 \pm 0.81$ & 0.024 & $3.13 \pm 1.25$ & $2.25 \pm 0.92$ & 0.031 \\
\hline Total cholesterol (mg/dl) & $210.09 \pm 39.46$ & $204.06 \pm 41.92$ & 0.002 & $204.80 \pm 37.98$ & $185.97 \pm 28.62$ & 0.017 \\
\hline LDL-C (mg/dl) & $129.20 \pm 33.11$ & $127.73 \pm 36.71$ & 0.004 & $123.54 \pm 30.33$ & $109.41 \pm 16.65$ & 0.094 \\
\hline $\mathrm{HDL}-\mathrm{C}(\mathrm{mg} / \mathrm{dl})$ & $49.17 \pm 10.56$ & $49.94 \pm 7.96$ & 0.046 & $49.34 \pm 11.29$ & $49.01 \pm 11.06$ & 0.138 \\
\hline TG (mg/dl) & $158.60 \pm 61.78$ & $137.84 \pm 61.55$ & 0.019 & $159.57 \pm 67.34$ & $132.93 \pm 53.95$ & 0.172 \\
\hline Creatinine clerance $\left(\mathrm{ml} / \mathrm{min} / 1.73 \mathrm{~m}^{2}\right)$ & $112.89 \pm 27.26$ & $123.36 \pm 31.44$ & 0.777 & $107.15 \pm 28.37$ & $121.75 \pm 39.10$ & 0.198 \\
\hline Cardiovascular risk score & $11.98 \pm 10.11$ & $7.37 \pm 4.48$ & 0.001 & $14.62 \pm 11.06$ & $8.05 \pm 7.72$ & 0.005 \\
\hline
\end{tabular}

Data are shown as mean \pm standard deviation (means \pm SD)

Abbreviations: BP blood pressure, $i P T H$ intact parathyroid hormone, 25(OH) vitamin D 25-hydroxyvitamin D3, HDL-C high-density-lipoprotein cholesterol, $L D L-C$ lowdensity-lipoprotein cholesterol, TG triglycerides, HOMA-IR homestasis model assessment-insulin resistance index, ALP alkaline phosphatase, PHPT primary hyperparathyroidism

Bold represents the significant $p$-values

not known. Hypercalcemic PHPT was reported to indicate an increased risk for cardiovascular diseases [19, 20]. Increased cardiovascular morbidity and mortality was reported in hypercalcemic PHPT [8-11]. Obesity [19], hypertension [8], hyperlipidemia [8], and diabetes [21] is higher in PHPT compared with the normal population, a potential cause for cardiovascular diseases [6, $22,23]$. Successful surgery for hyperparathyroidism is followed by improvements in hypertension, impaired glucose metabolism, dyslipidemia, and even in cardiovascular dysfunction [24, 25]. It is believed that elevated serum calcium and PTH concentrations might be directly or indirectly associated with increased cardiovascular risk [12-14]. However, there is still a lack of data on the potential cardiometabolic effects of normocalcemic PHPT after parathyroidectomy.

In present study, the prevalence of disease such as hypertension, diabetes, dyslipidemia, obesity, and insulin resistance were similarly increased in both patients with normocalcemic and hypercalcemic PHPT. Cardiometabolic risk factors including blood pressures, glucose metabolism and lipid profiles as well as cardiovascular risk score increased similarly in both patients with normocalcemic and hypercalcemic PHPT. Parathyroidectomy improved increased cardiovascular risk factors including systolic and diastolic BP, serum total cholesterol, and
HOMA-IR, as well as cardiovascular risk scores in both patients with hypercalcemic and normocalcemic PHPT. Ozturk et al. found similar metabolic disorders including hypertension and glucose intolerance in patients with normocalcemic and hypercalcemic PHPT [26]. Traditional cardiovascular risk factors including hypertension, hyperlipidemia, and impaired glucose tolerance were found to be similar in normocalcemic and hypercalcemic PHPT, whereas cardiovascular and cerebrovascular diseases were found higher in patients with hypercalcemic PHPT [16]. Although fasting blood glucose was found to be elevated in patients with normocalcemic PHPT, insulin resistance, lipid profiles, and blood pressures in those patients was similar to the controls $[27,28]$. Chen et al. observed that normocalcemic PHPT exhibited increased systolic and diastolic BP compared with the controls [29]. Increased PTH concentration was associated with increased fatal and non-fatal cardiovascular events [30]. Increased PTH was linked to increased cardiovascular morbidity and mortality, even in the absence of PHPT disease [20, 30, 31]. Increased PTH concentration was considered to be a potential marker of increased cardiovascular risk, even in the presence of normal calcium values [20,31].

In the present study, increased serum PTH concentration acted as a positive predictor of increased systolic 
Table 3 Metabolic parameters of subjects with and without fractures before and after parathyroidectomy

\begin{tabular}{|c|c|c|c|}
\hline & Subjects without fractures & Subjects with fractures & $\mathrm{p}$ \\
\hline \multicolumn{4}{|l|}{ Pre-parathyroidectomy } \\
\hline Cardiovascular risk score & $12.71 \pm 10.88$ & $17.85 \pm 7.04$ & 0.199 \\
\hline Diastolic BP (mmHg) & $81.93 \pm 6.29$ & $89.50 \pm 3.50$ & 0.001 \\
\hline Systolic BP (mmHg) & $137.37 \pm 16.61$ & $141.010 \pm 11.23$ & 0.551 \\
\hline iPTH $(\mathrm{pg} / \mathrm{ml})$ & $163.71 \pm 71.17$ & $194.01 \pm 98.26$ & 0.282 \\
\hline Calcium (mg/dl) & $10.62 \pm 0.77$ & $11.06 \pm 0.71$ & 0.136 \\
\hline Phosphorus (mg/dl) & $2.69 \pm 0.44$ & $2.42 \pm 0.49$ & 0.119 \\
\hline ALP (IU/L) & $98.76 \pm 27.35$ & $155.63 \pm 88.58$ & $<0.001$ \\
\hline Glucose (mg/dl) & $93.92 \pm 12.57$ & $95.13 \pm 20.42$ & 0.814 \\
\hline İnsulin ( $\mu \mid \mathrm{U} / \mathrm{ml})$ & $13.90 \pm 5.65$ & $8.57 \pm 3.49$ & 0.012 \\
\hline HOMA-IR & $3.22 \pm 1.33$ & $2.09 \pm 1.16$ & 0.026 \\
\hline Total cholesterol (mg/dl) & $206.67 \pm 39.21$ & $213.45 \pm 34.48$ & 0.643 \\
\hline LDL-C (mg/dl) & $126.19 \pm 32.69$ & $127.75 \pm 23.59$ & 0.897 \\
\hline $\mathrm{HDL}-\mathrm{C}(\mathrm{mg} / \mathrm{dl})$ & $49.63 \pm 11.04$ & $49.38 \pm 9.39$ & 0.429 \\
\hline $\mathrm{TG}(\mathrm{mg} / \mathrm{dl})$ & $155.95 \pm 57.35$ & $196.63 \pm 101.29$ & 0.092 \\
\hline \multicolumn{4}{|l|}{ Post-parathyroidectomy } \\
\hline Cardiovascular risk score & $6.72 \pm 4.92$ & $14.75 \pm 9.47$ & 0.010 \\
\hline Diastolic BP (mmHg) & $77.43 \pm 7.33$ & $85.00 \pm 6.48$ & 0.059 \\
\hline Systolic BP (mmHg) & $123.83 \pm 14.04$ & $121.51 \pm 25.43$ & 0.779 \\
\hline iPTH (pg/ml) & $61.46 \pm 23.57$ & $61.04 \pm 23.59$ & 0.971 \\
\hline Calcium (mg/dl) & $8.94 \pm 0.35$ & $9.06 \pm 0.44$ & 0.552 \\
\hline Phosphorus (mg/dl) & $3.26 \pm 0.36$ & $2.77 \pm 0.69$ & 0.034 \\
\hline ALP (IU/L) & $81.04 \pm 20.18$ & $121.56 \pm 27.24$ & 0.001 \\
\hline Glucose (mg/dl) & $85.40 \pm 6.01$ & $78.25 \pm 7.88$ & 0.038 \\
\hline İnsulin $(\mu \mathrm{IU} / \mathrm{ml})$ & $11.81 \pm 3.44$ & $6.53 \pm 3.26$ & 0.007 \\
\hline HOMA-IR & $2.55 \pm 0.80$ & $1.47 \pm 0.70$ & 0.015 \\
\hline Total cholesterol (mg/dl) & $194.49 \pm 37.62$ & $207.00 \pm 37.97$ & 0.537 \\
\hline $\mathrm{LDL}-\mathrm{C}(\mathrm{mg} / \mathrm{dl})$ & $119.70 \pm 32.10$ & $119.25 \pm 19.01$ & 0.978 \\
\hline $\mathrm{HDL}-\mathrm{C}(\mathrm{mg} / \mathrm{dl})$ & $49.30 \pm 9.23$ & $51.25 \pm 11.17$ & 0.757 \\
\hline $\mathrm{TG}(\mathrm{mg} / \mathrm{dl})$ & $133.36 \pm 54.56$ & $153.03 \pm 84.59$ & 0.530 \\
\hline
\end{tabular}

Data are shown as mean \pm standard deviation (means \pm SD)

Abbreviations: BP blood pressure, iPTH intact parathyroid hormone, 25(OH) vitamin D 25-hydroxyvitamin D3, HDL-C high-density-lipoprotein cholesterol, $L D L-C$ lowdensity-lipoprotein cholesterol, TG triglycerides, HOMA-IR homestasis model assessment-insulin resistance index, ALP alkaline phosphatase, PHPT primary hyperparathyroidism

Bold represents the significant $p$-values

and diastolic BP. Hagström et al. reported improvements in dyslipidemia following parathyroidectomy in asymptomatic mild PHPT [9]. A positive correlation between increased PTH level and hypertension was observed [32-35]. Increased serum PTH concentrations were reported as a predictor of increased systolic BP [32]. Parathyroidectomy improved increased systolic BP in mild PHPT [8]. Several mechanisms may explain the association between serum PTH and blood pressure. PTH might directly or indirectly cause increased aldosterone secretion by activating the renin-angiotensin system or it might cause increased vessel wall thickness by triggering the proliferation of vascular smooth muscle cells. The bidirectional interplay between aldosterone and PTH leads to an increased risk of developing metabolic and cardiovascular diseases [36]. Hyperparathyroidism is involved in increased cardiovascular risk; however, other factors including PTH receptors, cardiomyocytes [37], endothelial cells, and vascular smooth muscles are possibly involved in the increased cardiovascular risk.

Elevated PTH concentrations should also be considered beyond calcium and phosphate dysregulation when explaining cardiovascular diseases [30, 36]. Chronic PTH elevation was correlated with coronary microvascular 
dysfunction, cardiac hypertrophy, impaired glucose and lipid mechanisms, arterial hypertension, endothelial dysfunction, and subclinical aortic valve calcification $[1,20$, 38-40]. Such elevation might also be associated with pro-inflammatory protease and cytokine upregulation [30]. Elevated PTH concentrations were associated with adiposity and increased inflammatory cytokines in circulation (such as monocyte chemoattractant protein-1 and leptin) [40-42].

This case-control study and sample size was relatively small. These are limitations of this study.

\section{Conclusion}

The present study observed that normocalcemic PHPT and hypercalcemic PHPT were had similarly increased cardiovascular risk factors, even independent of serum calcium. The normocalcemic and hypercalcemic PHPT groups had similarly increased cardiovascular risk factors including blood pressures, glucose metabolism, and lipid profiles, as well as cardiovascular risk scores compared with controls. Parathyroidectomy ameliorated increased cardiovascular risk factors including blood pressures, total cholesterol, and HOMA-IR, as well as cardiovascular risk scores in patients with normocalcemic and hypercalcemic PHPT. Serum PTH acted as a positive predictor of increased blood pressures. Further studies are needed to examine the effect of parathyroidectomy on non-traditional manifestations of normocalcemic PHPT. Such studies will explain the potential mechanism between serum PTH and cardiometabolic disorders.

\section{Abbreviations}

25(OH) vitamin $D_{3}$ : 25-hydroxyvitamin $D_{3}$; ALP: Alkaline phosphatase; BP: Blood pressure; CRS: Cardiovascular risk score; HDL-C: High-densitylipoprotein cholesterol; HOMA-IR: Homeostasis model assessment-insulin resistance index; iPTH: Intact parathyroid hormone; LDL-C: Low-densitylipoprotein cholesterol; PHPT: Primary hyperparathyroidism; TG: Triglycerides

\section{Acknowledgements}

Not applicable.

\section{Funding}

No funding sources for research.

\section{Availability of data and materials}

All data are freely available for scientific purpose. The data can be found from authors.

\section{Authors' contributions}

$\mathrm{MC}$ and SB an, contributions to conception and design, or acquisition of data, or analysis and interpretation of data, involved in drafting the manuscript, MK, contributions to conception and design, or acquisition of data, or analysis and interpretation of data; MA, SK and MO, acquisition of data, or analysis and interpretation of data; EC, revising it critically for important intellectual content; and have given final approval of the version to be published. All authors read and approved the final manuscript.

\section{Ethics approval and consent to participate}

This retrospective study was approved by Diskapi Yildirim Beyazit Teaching and Research Hospital Ethics Board (No: 12.4.2015-25/04) and in written informed consent was obtained from the patients.
Consent for publication

Not applicable.

\section{Competing interests}

The authors declare that they have no competing interests.

\section{Publisher's Note}

Springer Nature remains neutral with regard to jurisdictional claims in published maps and institutional affiliations.

\section{Author details}

'Department of Endocrinology and Metabolism, Ankara Diskapi Yildirim Beyazit Teaching and Research Hospital, Ankara, Turkey. ${ }^{2}$ Department of Medical Biology, Baskent University, Ankara, Turkey. ${ }^{3}$ Department of Endocrinology and Metabolism, Afyonkarahisar Saglik Bilimleri University, Afyonkarahisar, Turkey.

Received: 12 October 2018 Accepted: 30 April 2019

Published online: 08 May 2019

\section{References}

1. Silverberg SJ, Clarke BL, Peacock M, Bandeira F, Boutroy S, et al. Current issues in the presentation of asymptomatic primary hyperparathyroidism: proceedings of the fourth international workshop. J Clin Endocrinol Metab. 2014:99:3580-94.

2. Díaz-Soto G, Julián MT, Puig-Domingo M. Normocalcemic primary hyperparathyroidism: a newly emerging disease needing therapeutic intervention. Hormones (Athens). 2012;11:390-6.

3. Bilezikian JP, Silverberg SJ. Normocalcemic primary hyperparathyroidism. Arq Bras Endocrinol Metabol. 2010;54:106-9.

4. Amaral LM, Queiroz DC, Marques TF, Mendes M, Bandeira F. Normocalcemic versus hypercalcemic primary hyperparathyroidism: more stone than bone? J Osteoporos. 2012;2012:128352

5. Cusano NE, Silverberg SJ, Bilezikian JP. Normocalcemic primary hyperparathyroidism. J Clin Densitom Off J Int Soc Clin Densitom. 2013;16:33-9.

6. Luboshitzky R, Chertok-Schaham Y, Lavi I, Ishay A. Cardiovascular risk factors in primary hyperparathyroidism. J Endocrinol Investig. 2009;32:317-21.

7. Tassone F, Maccario M, Gianotti L, Baffoni C, Pellegrino M, et al. Insulin sensitivity in normocalcaemic primary hyperparathyroidism. Endocrine. 2013;44:812-4.

8. Farahnak P, Lärfars G, Sten-Linder M, Nilsson I-L. Mild primary hyperparathyroidism: vitamin $\mathrm{D}$ deficiency and cardiovascular risk markers. $J$ Clin Endocrinol Metab. 2011:96:2112-8.

9. Hagström E, Lundgren $E$, Lithell $H$, Berglund L, Ljunghall S, et al. Normalized dyslipidaemia after parathyroidectomy in mild primary hyperparathyroidism: population-based study over five years. Clin Endocrinol. 2002;56:253-60.

10. Ishay A, Herer P, Luboshitzky R. Effects of successful parathyroidectomy on metabolic cardiovascular risk factors in patients with severe primary hyperparathyroidism. Endocr Pract Off J Am Coll Endocrinol Am Assoc Clin Endocrinol. 2011;17:584-90

11. Procopio M, Barale M, Bertaina S, Sigrist S, Mazzetti, et al. Cardiovascular risk and metabolic syndrome in primary hyperparathyroidism and their correlation to different clinical forms. Endocrine. 2014;47:581-9.

12. Ahlström T, Hagström E, Larsson A, Rudberg C, Lind L, et al. Correlation between plasma calcium, parathyroid hormone (PTH) and the metabolic syndrome (MetS) in a community-based cohort of men and women. Clin Endocrinol. 2009;71:673-8.

13. Hagström E, Michaëlsson $\mathrm{K}$. Melhus $\mathrm{H}$, Hansen T, Ahlström H, et al. Plasmaparathyroid hormone is associated with subclinical and clinical atherosclerotic disease in 2 community-based cohorts. Arterioscler Thromb Vasc Biol. 2014;34:1567-73.

14. Jorde R, Bonaa KH, Sundsfjord J. Population based study on serum ionised calcium, serum parathyroid hormone, and blood pressure. The Troms $\varnothing$ study. Eur J Endocrinol Eur Fed Endocr Soc. 1999;141:350-7.

15. Reid IR, Gamble GD, Bolland MJ. Circulating calcium concentrations, vascular disease and mortality: a systematic review. J Intern Med. 2016;279:524-40.

16. Tordjman KM, Yaron M, Izkhakov E, Osher E, Shenkerman G, et al. Cardiovascular risk factors and arterial rigidity are similar in asymptomatic normocalcemic and hypercalcemic primary hyperparathyroidism. Eur 」 Endocrinol Eur Fed Endocr Soc. 2010;162:925-33. 
17. Mather KJ, Hunt AE, Steinberg HO, Paradisi G, Hook G, et al. Repeatability characteristics of simple indices of insulin resistance: implications for research applications. J Clin Endocrinol Metab. 2001;86:5457-64.

18. Framingham, Heart study from: https://reference.medscape.com/calculator/ framingham-cardiovascular-disease-risk [viewed September 2014].

19. Bolland MJ, Grey AB, Gamble GD, Reid IR. Association between primary hyperparathyroidism and increased body weight: a meta-analysis. J Clin Endocrinol Metab. 2005;90:1525-30.

20. Kamycheva E, Sundsfjord J, Jorde R. Serum parathyroid hormone levels predict coronary heart disease: the Tromsø study. Eur J Cardiovasc Prev Rehabil Off J Eur Soc Cardiol Work Groups Epidemiol Prev Card Rehabil Exerc Physiol. 2004;11:69-74.

21. Reis JP, von Mühlen D, Kritz-Silverstein D, Wingard DL, Barrett-Connor E. Vitamin D, parathyroid hormone levels, and the prevalence of metabolic syndrome in community-dwelling older adults. Diabetes Care. 2007;30: 1549-55.

22. Han D, Trooskin S, Wang X. Prevalence of cardiovascular risk factors in male and female patients with primary hyperparathyroidism. J Endocrinol Investig. 2012;35:548-52.

23. Tassone F, Gianotti L, Baffoni C, Cesario F, Magro G, et al. Prevalence and characteristics of metabolic syndrome in primary hyperparathyroidism. J Endocrinol Investig. 2012;35:841-6.

24. Khaleeli AA, Johnson JN, Taylor WH. Prevalence of glucose intolerance in primary hyperparathyroidism and the benefit of parathyroidectomy. Diabetes Metab Res Rev. 2007;23:43-8.

25. Nilsson I-L, Aberg J, Rastad J, Lind L. Maintained normalization of cardiovascular dysfunction 5 years after parathyroidectomy in primary hyperparathyroidism. Surgery. 2005;137:632-8.

26. Yener Ozturk F, Erol S, Canat MM, Karatas S, Kuzu I, et al. Patients with normocalcemic primary hyperparathyroidism may have similar metabolic profile as hypercalcemic patients. Endocr J. 2016;63:111-8.

27. Temizkan S, Kocak O, Aydin K, Ozderya A, Arslan G, et al. Normocalcemic hyperparathyroidism and insulin resistance. Endocr Pract Off J Am Coll Endocrinol Am Assoc Clin Endocrinol. 2015:21:23-9.

28. Hagström E, Lundgren E, Rastad J, Hellman P. Metabolic abnormalities in patients with normocalcemic hyperparathyroidism detected at a populationbased screening. Eur J Endocrinol Eur Fed Endocr Soc. 2006;155:33-9.

29. Chen G, Xue Y, Zhang Q, Xue T, Yao J, et al. Is Normocalcemic primary hyperparathyroidism harmful or harmless? J Clin Endocrinol Metab. 2015; 100:2420-4.

30. Van Ballegooijen AJ, Reinders I, Visser M, Brouwer IA. Parathyroid hormone and cardiovascular disease events: A systematic review and meta-analysis of prospective studies. Am Heart J. 2013;165(655-664):664.e1-5.

31. Hagström E, Hellman $P$, Larsson TE, Ingelsson E, Berglund L, et al. Plasma parathyroid hormone and the risk of cardiovascular mortality in the community. Circulation. 2009;119:2765-71.

32. Jorde R, Svartberg J, Sundsfjord J. Serum parathyroid hormone as a predictor of increase in systolic blood pressure in men. J Hypertens. 2005;23:1639-44.

33. Lee DM, Rutter MK, O'Neill TW, Boonen S, Vanderschueren D, et al. Vitamin D, parathyroid hormone and the metabolic syndrome in middle-aged and older European men. Eur J Endocrinol Eur Fed Endocr Soc. 2009;161:947-54.

34. Snijder MB, Lips P, Seidell JC, Visser M, Deeg DJH, et al. Vitamin D status and parathyroid hormone levels in relation to blood pressure: a populationbased study in older men and women. J Intern Med. 2007;261:558-65.

35. Hjelmesaeth J, Hofsø D, Aasheim ET, Jenssen T, Moan J, et al. Parathyroid hormone, but not vitamin $\mathrm{D}$, is associated with the metabolic syndrome in morbidly obese women and men: a cross-sectional study. Cardiovasc Diabetol. 2009;8:7

36. Tomaschitz A, Ritz E, Pieske B, Rus-Machan J, Kienreich K, et al. Aldosterone and parathyroid hormone interactions as mediators of metabolic and cardiovascular disease. Metabolism. 2014;63:20-31.

37. Potthoff SA, Janus $A$, Hoch $H$, Frahnert M, Tossios P, et al. PTH-receptors regulate norepinephrine release in human heart and kidney. Regul Pept. 2011;171:35-42.
38. Osto E, Fallo F, Pelizzo MR, Maddalozzo A, Sorgato N, et al. Coronary microvascular dysfunction induced by primary hyperparathyroidism is restored after parathyroidectomy. Circulation. 2012;126:1031-9.

39. Almqvist EG, Bondeson A-G, Bondeson L, Svensson J. Increased markers of inflammation and endothelial dysfunction in patients with mild primary hyperparathyroidism. Scand J Clin Lab Invest. 2011;71:139-44.

40. Iwata S, Walker MD, Di Tullio MR, Hyodo E, Jin Z, et al. Aortic valve calcification in mild primary hyperparathyroidism. J Clin Endocrinol Metab. 2012:97:132-7.

41. Delfini E, Petramala L, Caliumi C, Cotesta D, De Toma G, et al. Circulating leptin and adiponectin levels in patients with primary hyperparathyroidism. Metabolism. 2007:56:30-6.

42. Sukumar D, Partridge NC, Wang X, Shapses SA. The high serum monocyte chemoattractant protein-1 in obesity is influenced by high parathyroid hormone and not adiposity. J Clin Endocrinol Metab. 2011:96:1852-8.

\section{Ready to submit your research? Choose BMC and benefit from:}

- fast, convenient online submission

- thorough peer review by experienced researchers in your field

- rapid publication on acceptance

- support for research data, including large and complex data types

- gold Open Access which fosters wider collaboration and increased citations

- maximum visibility for your research: over $100 \mathrm{M}$ website views per year

At $\mathrm{BMC}$, research is always in progress.

Learn more biomedcentral.com/submissions 